\title{
Irrational Economic Decision-Making after Ventromedial Prefrontal Damage: Evidence from the Ultimatum Game
}

\author{
Michael Koenigs and Daniel Tranel \\ Department of Neurology, Division of Cognitive Neuroscience, University of Iowa College of Medicine and Neuroscience Graduate Program, University of \\ Iowa, Iowa City, Iowa 52242
}

\begin{abstract}
Emotion regulation is often critical for adaptive decision making. Here, we investigate whether emotion regulation defects following focal prefrontal brain damage are associated with exceptionally irrational economic decision making in situations of unfair treatment. In the Ultimatum Game, two players are given one opportunity to split a sum of money. One player (the proposer) offers a portion of the money to the second player (the responder) and keeps the rest. The responder can either accept the offer (in which case both players split the money as proposed) or reject the offer (in which case both players get nothing). Relatively low Ultimatum offers are often rejected, and this "irrational" behavior has been attributed to an emotional reaction to unfair treatment. Using the lesion method, we tested the hypothesis that damage to ventromedial prefrontal cortex (VMPC), an area critical for the modulation of emotional reactions, would result in exaggerated irrational economic decisions. Subjects acted as the responder to 22 different proposers who offered various splits of $\$ 10$. Offers ranged from fair (give \$5, keep \$5) to extremely unfair (give \$1, keep \$9). The rejection rate of the VMPC group was higher than the rejection rates of the comparison groups for each of the most unfair offers $(\$ 7 / \$ 3, \$ 8 / \$ 2, \$ 9 / \$ 1)$. These results suggest that emotion regulation processes subserved by VMPC are a critical component of normal economic decision making.
\end{abstract}

Key words: decision making; emotion; human; lesion; neuroeconomics; orbitofrontal; prefrontal; ventromedial

\section{Introduction}

The ability to regulate emotion is critical for adaptive human behavior. Recent functional imaging work implicates areas of prefrontal cortex in the volitional control of emotion (Beauregard et al., 2001; Ochsner et al., 2002, 2004; Levesque et al., 2003; Phan et al., 2005). Patients with acquired defects in emotion regulation after focal prefrontal brain injury afford a unique opportunity to study how emotion regulation processes may contribute to decision making. Ventromedial prefrontal cortex (VMPC) damage is reliably associated with defective emotion modulation; specifically, poorly controlled emotional responses that are incommensurate with the precipitating event. Clinical descriptions of patients with acquired VMPC damage consistently indicate that despite generally blunted affect, in response to relatively minor provocation or frustration, such patients may be shorttempered, irritable, angry, argumentative, and even abusive (Burgess and Wood, 1990; Grafman et al., 1996; Barrash et al., 2000; Berlin et al., 2004; Anderson et al., 2006). For the current study, we assembled a group of seven patients with bilateral VMPC damage, all of whom have evinced marked emotion regulation defects after their brain injuries (Fig. 1, Table 1).

Although emotion regulation impairment after VMPC dam-

Received July 26, 2006; revised Dec. 9, 2006; accepted Dec. 18, 2006.

This work was supported by National Institute of Neurological Disorders and Stroke Grant P01 NS19632 and National Institute on Drug Abuse Grant R01 DA022549. We thank Hanna Damasio for making available her neuroanatomical analyses of VMPC patients and for preparing Figure 1.

Correspondence should be addressed to Dr. Michael Koenigs, Building 10, Room 7-5648, 10 Center Drive, Bethesda, MD 20892. E-mail: koenigsm@ninds.nih.gov.

DOI:10.1523/JNEUROSCI.4606-06.2007

Copyright $\odot 2007$ Society for Neuroscience $\quad$ 0270-6474/07/270951-06\$15.00/0 age is a robust clinical finding, the potential impact of this phenomenon on distinct domains of decision making has not been explored systematically in the laboratory. Here, we report data indicating that VMPC is critical for normal economic decision making in circumstances of social frustration.

The Ultimatum Game is a widely used laboratory model of economic decision making. In a typical instantiation, two players are given one opportunity to split a sum of money. One player (the proposer) offers a portion of the money to the second player (the responder). The responder can either accept the offer (in which case both players split the money as proposed) or reject the offer (in which case both players get nothing). "Rational actor" models predict that the responder would accept any offer, no matter how low. However, relatively small offers $(\sim 20 \%$ of the total) have a $\sim 50 \%$ chance of being rejected (Guth et al., 1982; Bolton and Zwick, 1995). The "irrational" rejection of unfair Ultimatum Game offers has been correlated with feelings of anger (Pillutla and Murnighan, 1996), increased skin conductance responses (van't Wout et al., 2006), and activation of the insula (Sanfey et al., 2003), a brain area associated with negative emotional states (Phillips et al., 1997; Calder et al., 2000; Damasio et al., 2000). These results converge to suggest that the irrational rejection of Ultimatum offers in normal individuals is driven by an emotional response to unfair treatment. The regulation of emotion may therefore be essential for "rational" acceptance of unfair offers, and consequently VMPC may be critical for normal decision making in this context.

To investigate this possibility, we conducted a study in which VMPC patients with documented impairments in emotion regulation acted as responders in the Ultimatum Game. We hypoth- 
esized that for unfair offers, the acceptance rate of VMPC patients would be abnormally low (i.e., hyper-irrational) compared with brain-damaged and healthy comparison groups.

\section{Materials and Methods}

Participants. The "target" participants were seven patients with adult-onset bilateral VMPC damage (see Fig. 1 for lesion overlap). The target patients, for the most part, have intact psychometric intelligence, memory, and executive function, but they all have documented impairments in social and emotional functioning (Table 1) (supplemental material, available at www.jneurosci.org). In particular, every target patient has a documented defect in the regulation of emotion. Fourteen patients with adultonset lesions outside of VMPC [brain-damaged comparison group (BDC)] and 14 neurologically normal adults [normal comparison group (NC)] also participated. The VMPC, BDC, and $\mathrm{NC}$ groups were matched for age and sex distribution (Table 2) (see supplemental material for demographic and background data, available at www.jneurosci.org).

VMPC patients were selected on the basis of having damage that included VMPC in both hemispheres, where VMPC is defined as the medial one-third of the orbital surface and the ventral one-third of medial surface of prefrontal cortex. In the VMPC group, the lesions were caused by meningioma resection (three cases) or subarachnoid hemorrhage after rupture of anterior communicating artery aneurysm (four cases). The BDC patients were selected on the basis of having damage that did not encroach into VMPC in either hemisphere, and that also spared the amygdala and insula in both hemispheres [the amygdala and insular cortices have been implicated in emotional processing (for review, see Bechara, 2004)]. In the BDC group, the lesions were caused by cerebrovascular accident in eight patients, by meningioma resection in five patients, and by surgical treatment of a subdural hematoma in one patient. In nine of the BDC patients, the lesion was in the left hemisphere, in either the lateral temporal (four patients), dorsolateral prefrontal (three patients), superior mesial prefrontal (one patient), or thalamic (one patient) regions; in four BDC patients, the lesion was in the right hemisphere, in either the dorsolateral prefrontal (two patients) or lateral temporal (two patients) region; one BDC patient had a bilateral lesion in the superior mesial prefrontal region. The lesions in the BDC patients varied in size, with some being larger than any of the VMPC cases, and some being smaller.

Patients were selected from the Patient Registry of the Division of Cognitive Neuroscience at the University of Iowa (Iowa City, IA). All patients conformed to the inclusion criteria of the Patient Registry. They had focal, stable lesions that could be clearly identified on magnetic resonance (MR) or computerized tomography (CT) scans, and they were free of dementia, psychiatric disorder, and substance abuse. All participants were free of (VMPC, BDC)
Table 1. Social/emotional function and neuropsychological data as a function of patient (VMPC group) or group

\begin{tabular}{lllrclcc}
\hline Patient & Acquired sociopathy $^{a}$ & Regulation defect $^{b}$ & IQ & WMI & WCST Cat. & WCST PE & TMT B - A \\
\hline 1 & Yes (3) & Yes (3) & 143 & 124 & 6 & 4 & 37 \\
2 & Yes (3) & Yes (3) & 108 & 118 & 6 & 7 & 82 \\
3 & Yes (3) & Yes (3) & 91 & 102 & 6 & 8 & 71 \\
4 & Yes (3) & Yes (3) & 104 & 105 & 6 & 10 & 27 \\
5 & Yes (3) & Yes (2) & 106 & 124 & 6 & 9 & 13 \\
6 & Yes (2) & Yes (2) & 109 & 102 & 6 & 7 & 21 \\
7 & Yes (3) & Yes (3) & 84 & 88 & 0 & 66 & 126 \\
VMPC mean & Yes (2.9) & Yes (2.7) & 106.4 & 109.0 & 5.1 & $\frac{66}{15} .9$ & 53.9 \\
(SD) & (0.4) & $(0.5)$ & $(18.7)$ & $(13.5)$ & $(2.3)$ & $(22.2)$ & $(40.9)$ \\
BDC mean & No (0) & No (0) & 99.8 & 97.9 & 4.0 & 16.4 & 52.4 \\
(SD) & & & $(15.3)$ & $(14.2)$ & $(2.4)$ & $(13.3)$ & $(39.0)$ \\
\hline
\end{tabular}

The numbers in parentheses denote degree of severity, where 1 is mild, 2 is moderate, and 3 is severe. All BDC patients were normal on these measures. I0 Wechsler Adult Intelligence Scale-III (WAIS-III) Full Scale IQ; WMI, Wechsler Memory Scale-III (WMS-III) Working Memory Index. For the WAIS-III and WMS-III, scores are standard scores, where the mean is 100 and the SD is 15. WCST, Wisconsin Card Sorting Test (Cat., number of categories completed; PE, number of perseverative errors); TMT, Trailmaking Test (B - A, Part B minus Part A times to completion, in seconds; higher scores are worse). Underlined scores are defective. One VMPC patient (7) had significant defects on most of the executive functioning measures; the other six were nearly flawless across the board, and as a group, the VMPC patients did not differ from the BDC patients on any of the measures reported here ( $p$ values $>0.10$ in $t$ test). Additional neuropsychological data are provided in the supplemental material (available at www.jneurosci.org).

${ }^{a}$ Acquired sociopathy refers to whether the participant met criteria for acquired sociopathy.

${ }^{b}$ Regulation defect refers to whether the participant evinces poorly modulated emotional reactions (lability, irritability, tantrums, outbursts). The ratings for acquired sociopathy and regulation defect were derived from lowa Rating Scales of Personality Change and from interviews and medical records.
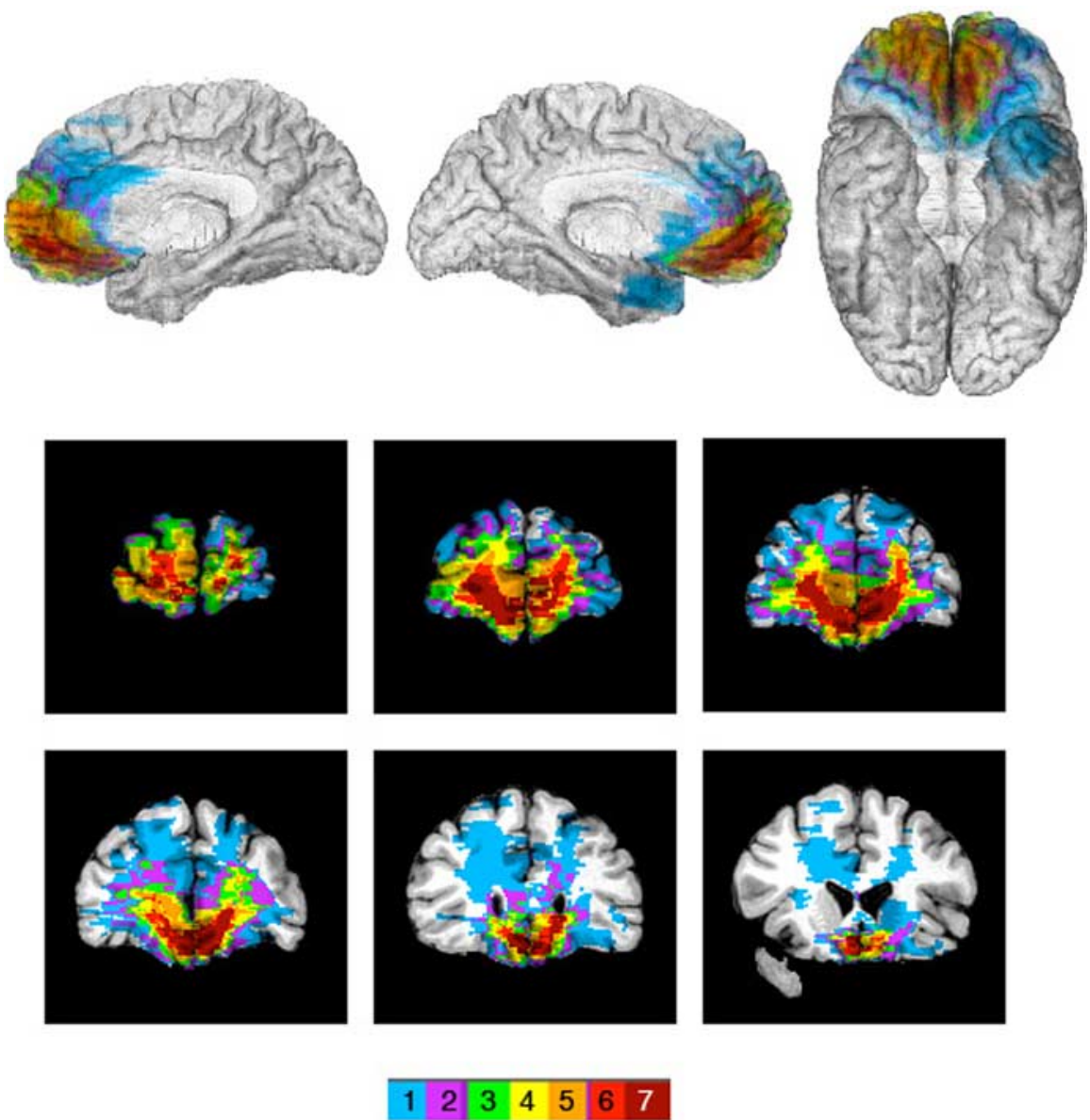

\# of overlaps

Figure 1. Lesion overlap of VMPC patients. Lesions of the seven VMPC patients displayed in ventral and mesial views and coronal slices. The color bar indicates the number of overlapping lesions at each voxel. Maximal overlap occurs in the mesial and anterior portions of orbitofronal cortex as well as the ventral portions of mesial prefrontal cortex (Brodmann areas 10, 11, 25, and subgenual 32). VMPC refers to this area of maximal lesion overlap.

significant intellectual impairments. The patients had no premorbid histories of abnormal social conduct, emotional maladjustment, or other psychological disturbance. Neuropsychological, neuroanatomical, and experimental studies were all conducted in the chronic phase of recovery, 
Table 2. Demographic and background data as a function of patient (VMPC group) or group (VMPC, BDC, NC)

\begin{tabular}{lllll}
\hline Patient & Age & Educ. & Sex & Etiology \\
\hline 1 & 64 & 14 & $\mathrm{M}$ & Meningioma resection \\
2 & 64 & 16 & $\mathrm{~F}$ & Meningioma resection \\
3 & 62 & 8 & $\mathrm{M}$ & SAH; ACoA aneurysm \\
4 & 42 & 13 & $\mathrm{~F}$ & SAH; ACoA aneurysm \\
5 & 57 & 14 & $\mathrm{~F}$ & SAH; ACoA aneurysm \\
6 & 58 & 13 & $\mathrm{~F}$ & Meningioma resection \\
7 & 65 & 11 & $\mathrm{M}$ & SAH; ACoA aneurysm \\
VMPC mean & 58.9 & 12.7 & $3 \mathrm{M} / 4 \mathrm{~F}$ & \\
SD & $(8.1)$ & $(2.6)$ & $7 \mathrm{M} / 7 \mathrm{~F}$ & \\
BDC mean & 14.0 & $6 \mathrm{M} / 8 \mathrm{~F}$ & \\
SD & 57.2 & $(2.4)$ & & \\
NC mean & $(10.7)$ & $\mathrm{n} / \mathrm{a}$ & & \\
SD & 57.6 & & & \\
\hline
\end{tabular}

Age, Age of participant at time of Ultimatum Game testing, in years; Educ., years of education completed; SAH, subarachnoid hemorrhage; ACoA, anterio communicating artery; $\mathrm{n} / \mathrm{a}$, data not available; $\mathrm{M}$, male; $\mathrm{F}$, female. The average age did not differ among groups (one-way ANOVA; $p=0.84$ ), and average education did not differ between the VMPC and BDC groups ( $t$ test; $p=0.27$ ). Additional background data are provided in the supplemental material (available at www.jneurosci.org).

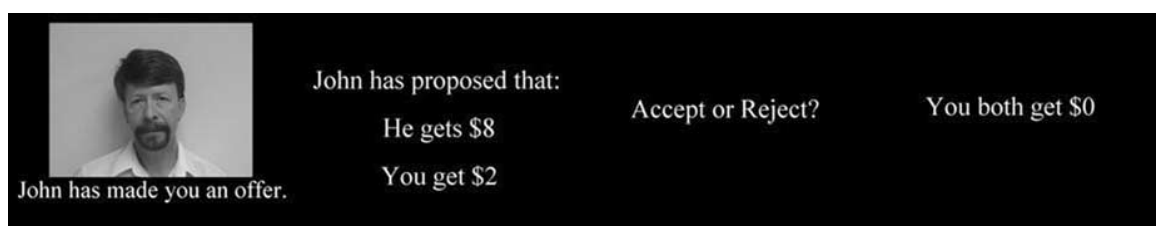

Figure 2. Schematic of trial design. First column, Proposer name/picture: 4 s. Second column, 0ffer: $4 \mathrm{~s}$. Third column, Subject decision: indefinite. Fourth column, Outcome: $3 \mathrm{~s}$. In this example, the offer was rejected.

more than 3 months after lesion onset. All lesions were acquired in adulthood. Normal participants were recruited from the surrounding community through advertisement, and they were compensated for their participation. All participants gave informed consent before completing the study, which was approved by the Human Subjects Committee of the University of Iowa.

Lesion analysis. The neuroanatomical analysis (Fig. 1) was based on MR data for three subjects (those with lesions resulting from surgical resection of an orbital meningioma) and CT data for four subjects (those with lesions resulting from subarachnoid hemorrhage subsequent to rupture of an anterior communicating artery aneurysm). All neuroimaging data were obtained in the chronic epoch. Each patient's lesion was reconstructed in three dimensions using Brainvox (Damasio and Frank, 1992; Frank et al., 1997). Using the MAP-3 technique (Damasio et al., 2004), the lesion contour for each patient was manually warped into a normal template brain. The overlap of lesions in this volume, calculated by the sum of $n$ lesions overlapping on any single voxel, is color coded.

Task. Participants acted as responders in a series of 22 trials of the Ultimatum Game. In each trial, the participant first saw a picture of a person making an Ultimatum offer (some take-it-or-leave-it split of \$10) with the person's name. Next, the participant saw the offer (e.g., "John gets $\$ 8$, you get $\$ 2$ "). Then, the participant saw “Accept or Reject?” on the screen. The participant had unlimited time to consider the offer and push a button ("Accept" or "Reject") to respond. ${ }^{a}$ Last, the participant saw the outcome based on his/her response (e.g., "You both get $\$ 0$ " if the offer was rejected or "You get \$2" if the offer was accepted) (see Fig. 2 for schematic of trial design). The intertrial interval (fixation cross) was $3 \mathrm{~s}$. Participants received 22 offers from 22 different proposers. Before beginning, the participants were instructed about the contingencies of an "Accept" or "Reject" response, that the offers were real but made before their arrival, that the participant's responses would not affect subsequent offers, and that both the participant and the proposer would be paid according to the participant's decisions. In fact, the 22 pictured "propos-

${ }^{a}$ Because the offer screen was presented for a fixed $4 \mathrm{~s}$ before the "Accept or Reject?" screen appeared, and some subjects would attempt to make their response before the appearance of that screen whereas others would wait, response times for the "Accept or Reject?" screen were not meaningful, and thus response times are not reported. ers" were confederates of the experimenter, and the offers were predetermined by the experimenter. In compliance with the University of Iowa Institutional Review Board, all participants were paid the same amount, regardless of their responses on the task. At the end of the testing session, participants underwent debriefing to explain this requirement.

Offers. All participants received the same 22 offers in fixed random order. Because the participants' responses to unfair offers were of the greatest interest, offers were generated in the following frequencies: two offers of $\$ 5$ (proposer keeps $\$ 5$ ), two offers of $\$ 4$ (proposer keeps $\$ 6$ ), six offers of $\$ 3$ (proposer keeps $\$ 7$ ), six offers of $\$ 2$ (proposer keeps $\$ 8$ ), and six offers of $\$ 1$ (proposer keeps $\$ 9$ ).

For the purposes of this study, we considered the $\$ 5$ and $\$ 4$ offers to be fair and the $\$ 3, \$ 2$, and $\$ 1$ offers to be unfair. The justification for this was based on two lines of empirical data. For one, a separate group of 10 neurologically normal adults was instructed on the rules of the Ultimatum Game and asked to make a subjective judgment of each offer amount as fair or unfair. The $\$ 5$ and $\$ 4$ offers were judged to be fair in 10 of 10 and 9 of 10 cases, respectively, whereas $\$ 3, \$ 2$, and $\$ 1$ offers were judged to be fair in only 3 of 10,1 of 10 , and 1 of 10 cases, respectively. The modal response (given by 6 of 10 respondents) was that $\$ 5$ and $\$ 4$ offers were fair, but $\$ 3, \$ 2$, and $\$ 1$ offers were unfair. Second, in a previous study of Ultimatum Game responses, it was found that offers of $35 \%$ of the total sum and greater (corresponding to the $\$ 4$ and $\$ 5$ offers in this experiment) were virtually unanimously accepted, whereas lesser offers (corresponding to the $\$ 3, \$ 2$, and $\$ 1$ offers in this experiment) were rejected a significant proportion of the time (Guth et al., 1982). These two sources of data support our operationalization of fair ( $\$ 5$ and $\$ 4$ ) and unfair $(\$ 3, \$ 2$, and $\$ 1)$ offers.

Manipulation check. After the offers, but before debriefing, the participant answered the following two questions: (1) "Did you believe that the offers were made by the pictured people?" and (2) "Did you believe that you and the proposer would actually be paid according to your decisions?" Participants responded by circling a number on a seven-point Likert-type scale: -3 corresponded to "not at all," 0 corresponded to "no idea," and + 3 corresponded to "completely." Participants endorsing a negative number on either question were excluded. None of the seven VMPC patients were excluded on this basis. Three BDC subjects and four NC subjects were excluded, so additional participants were recruited to reach the final number of 14 in each comparison group. The foregoing participant data apply to the final groups (after exclusion and replacement). The proportion of excluded participants did not differ between groups $\left(\chi^{2}=2.41 ; p=0.30\right)$.

Data analysis. To test for between-group differences in the probability of accepting unfair offers, we used a logistic regression fitted with the generalized estimating equations (GEE) method. The logistic regression fitted with the GEE method is a nonparametric test of response probability that accounts for the correlation of responses within individual subjects for a given offer amount (Hanley et al., 2003). This analysis generates an estimate of odds ratio, $95 \%$ confidence interval, and an associated $p$ value. All $p$ values are corrected for multiple comparisons.

\section{Results}

As expected, all three groups unanimously accepted the relatively fair ( $\$ 5$ and $\$ 4$ ) offers but rejected a significant proportion of unfair offers. The NC and BDC groups exhibited similar acceptance rates for unfair offers: 85 and $94 \%$, respectively, for $\$ 3$ offers $(p=0.60), 55$ and $50 \%$ for $\$ 2$ offers, respectively $(p>$ 


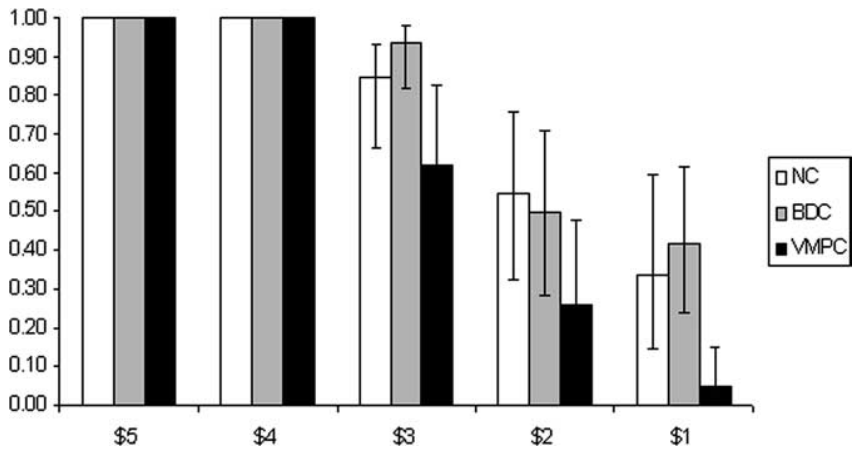

Figure 3. Group acceptance rates of Ultimatum Game offers. The bars represent the proportion of accepted offers ( $y$-axis) for each offer amount ( $x$-axis) for each group (legend). Error bars indicate $95 \%$ confidence intervals for the probability of acceptance. The VMPC group's acceptance rate was lower than that of the $\mathrm{NC}$ and BDC groups for the $\$ 3$ offers $(p=0.33$ and $p=$ 0.022 , respectively), $\$ 2$ offers $(p=0.22$ and $p=0.37$, respectively), and $\$ 1$ offers $(p=0.017$ and $p=0.0015$, respectively).

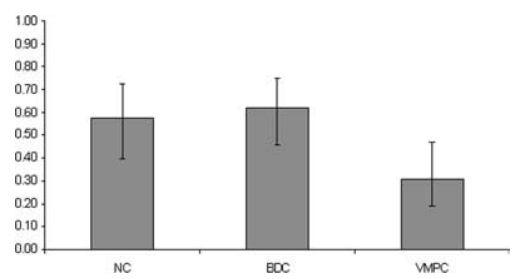

Figure 4. Overall group acceptance rates of unfair $(\$ 3, \$ 2$, and $\$ 1)$ offers. The bars represent the proportion of accepted offers ( $y$-axis) for each subject group ( $x$-axis). Error bars indicate $95 \%$ confidence intervals for the probability of acceptance. Across all unfair offers, the VMPC group's acceptance rate was lower than those of the NC group $(p=0.081)$ and BDC group $(p=0.020)$.

$0.99)$, and 33 and $42 \%$ for $\$ 1$ offers, respectively $(p>0.99)$. In contrast, patients with bilateral VMPC damage accepted a smaller proportion of each level of unfair offer $(62,26$, and $5 \%$ for $\$ 3, \$ 2$, and $\$ 1$ offers, respectively) than the NC group ( $p=0.33$, $p=0.22$, and $p=0.017$ for $\$ 3, \$ 2$, and $\$ 1$, respectively) or the BDC group $(p=0.022, p=0.37$, and $p=0.0015$ for $\$ 3, \$ 2$, and $\$ 1$, respectively) (Fig. 3). Across all three levels of unfair offers $(\$ 3, \$ 2$, and $\$ 1)$, the NC and BDC groups accepted 58 and $62 \%$, respectively ( $p>0.99$ ), whereas the VMPC group accepted only $31 \%(p=0.081$ and $p=0.020$ compared with $\mathrm{NC}$ and BDC groups, respectively) (Fig. 4).

In summary, $\mathrm{NC}$ and BDC groups accepted similar proportions of unfair offers, whereas the acceptance rate of the VMPC group was consistently lower for unfair offers. In every case where group differences were statistically significant ( $\$ 3$ offers, $\$ 1$ offers, and overall unfair offers), the VMPC group had a lower rate of acceptance than at least one comparison group. For all three levels of unfair offers, the groups' acceptance response pattern was the same: $\mathrm{NC}=\mathrm{BDC}>\mathrm{VMPC}$.

\section{Discussion}

The crux of the Ultimatum decision is the conflict between accepting unfair treatment and foregoing financial gain. Psychological, psychophysiological, and functional imaging data indicate that a negative emotional response to unfair treatment is associated with the normal, albeit irrational, rejections of low Ultimatum Game offers (Pillutla and Murnighan, 1996; Sanfey et al., 2003; v'ant Wout et al., 2006), consistent with the notion that the modulation of emotional response is critically involved in the responder's decision-making process. Here, we show that dam- age to VMPC, an area necessary for the normal regulation of emotion, affects economic decision making. The hyper-irrational rejection of unfair Ultimatum offers observed in the VMPC patients therefore supports the view that emotion regulation is a fundamental component of normal economic decision making in situations where financial considerations conflict with emotional responses. It is important to situate this result in the context of previous work on emotion, decision making, and VMPC, as well as discuss alternate explanations of the observed effect.

One of the most robust clinical findings associated with VMPC damage is the defective modulation of emotion. As discussed previously, patients with VMPC damage tend to exhibit exaggerated anger, irritability, emotional outbursts, and tantrums, particularly in social situations involving frustration or provocation (Burgess and Wood, 1990; Grafman et al., 1996; Barrash et al., 2000; Berlin et al., 2004; Anderson et al., 2006). All seven VMPC patients who participated in this study have demonstrated such behavior in their personal lives. ${ }^{b}$ In light of this behavioral attribute, the hyper-irrational behavior of VMPC patients in our study can be explained by the circumstances of the Ultimatum Game: situations of provocation/frustration arise (unfair treatment by another individual), and anger is the normal predominant reaction. In such circumstances, the modulation of emotional reaction is essential for financially advantageous decision making, and this is precisely where VMPC patients diverge from comparison groups. Normative behavioral data also support this interpretation: Kravitz and Gunto (1992) found that in normal subjects, rejection rates increased when small Ultimatum offers were accompanied by provocation or insult (e.g., "I know you'd like more, but that's the way it goes. Take it or leave it.").

However, because the current study was not designed to obtain a direct measure of subjects' emotional responses during performance of the Ultimatum Game task (e.g., subjective ratings or psychophysiological recording), our conclusions about the specific role of emotion in Ultimatum game responding are inferred based on the target patients' behavioral characteristics as well as convergent psychological and neurophysiological data regarding the Ultimatum Game. Although we did observe some VMPC participants to have strong negative emotional reactions to unfair offers, collection of direct, systematic measurements of emotional responses is an important next step in this line of work. Based on what we have seen thus far, we would expect measurements of subjective feeling to document negative emotional "overreactions" in the VMPC patients.

Despite the convergent lines of evidence implicating emotion regulation defects as the likely basis of the observed results, alternative explanations warrant consideration. Conventional neuropsychological data argue against deficits in cognitive function playing a significant role in the observed results: VMPC and BDC groups did not differ in measures of general intelligence (WAISIII Verbal IQ, Performance IQ, Full Scale IQ, and estimated premorbid Full Scale IQ), memory (WMS-III General Memory Index and Working Memory Index), or executive function (Wisconsin Card Sort Test and Trailmaking Test), nor did they differ on demographic variables (Tables 1,2) (supplemental material, available at www.jneurosci.org). However, experimental

${ }^{b}$ For example, one of the target patients (patient 2) is accompanied to her hospital visits by her nearly 90 -year-old mother. In a recent visit to our neurology clinic, the mother was slow to find that patient's appointment slip during their arrival. Patient 2 reacted by screaming violently at her mother in the patient waiting area. Other patients have similar tantrum-like responses to relatively minor provocation. Examples of triggers for similar outbursts of anger include political disagreements with friends (Patient 3), a spouse's suggestion to use headlights while driving (Patient 1), and a mother's recommendation to change a clothing combination (Patient 4). The key feature of the target patients' angry responses towards others is that they are not commensurate with the provocation. 
data indicate that areas within and adjacent to VMPC are important for the representation of reward as well as flexibility in decision making as reward contingencies change (Rolls et al., 1994; O'Doherty et al., 2001). Perhaps deficits related to reward processing contribute to the VMPC patients' abnormal pattern of responses. One possibility is that the VMPC patients' high rejection rate is more a result of differences in sensitivity to financial outcome than to emotional reaction to unfair treatment. In a task involving a choice between two risky gambles, VMPC patients exhibited abnormally low levels of autonomic arousal and reported abnormally low levels of subjective sadness when the nonchosen gamble was revealed to be of greater reward than the chosen gamble (Camille et al., 2004). If this lack of emotional reaction to a better possible financial outcome (so-called "lack of regret") in VMPC patients holds in the Ultimatum Game, then the anticipated regret of foregoing financial gain may not have the same impact on decision making in the VMPC patients as in normal individuals. That is, if the emotional reaction against not obtaining the offered money is diminished, VMPC patients may find it easier to reject the offer. However, at least two studies report that VMPC patients have normal emotional responses to simple financial gain and loss (Bechara et al., 1996; Camille et al., 2004), suggesting that VMPC patients do not have diminished sensitivity to financial consequences, per se. Although differences in reward processing or sensitivity to financial outcomes may contribute to the VMPC group's Ultimatum Game abnormal responses, poorly modulated emotion is, in our view, more likely the primary factor. Future work is required to fully resolve this issue.

It is important to note that previous work regarding the relationship among VMPC, emotion, and decision making has focused on the hypo-emotionality of VMPC patients. In fact, outside the aforementioned irritability and emotion modulation defects, VMPC patients have often been noted to have blunted or flattened affect (Blumer and Benson, 1975; Eslinger and Damasio, 1985; Shallice and Burgess, 1991; Barrash et al., 2000). In laboratory studies, VMPC patients exhibit diminished autonomic arousal and subjective feeling in response to emotionally charged pictures (Damasio et al., 1990; Blair and Cipolotti, 2000) and emotional memories (Tranel et al., 1998). Also in line with these results, at least three experimental studies have demonstrated abnormal economic decision making in VMPC patients that has been attributed to hypo-emotionality (Bechara et al., 1996; Camille et al., 2004; Shiv et al., 2005). However, the tasks used in these studies differ from the Ultimatum Game in a fundamental way: in all three studies, the tasks include, as their primary feature, a gamble. In contrast, in the Ultimatum Game the exact contingencies of the responder's decisions are always known to the responder: there is no gamble. Consequently, the emotional component of the Ultimatum Game seems to be fundamentally different from the gambling games: whereas in the gambling games, the emotion is derived from the risk and uncertainty associated with the gamble, in the Ultimatum Game the emotion is derived from the unfair treatment by the proposer. Thus, the Ultimatum Game allows one to examine a heretofore unexplored effect of VMPC damage on decision making, specifically the poorly modulated emotional reaction to social frustration. Although this modulation defect has been commonly reported as part of the clinical profile of VMPC patients, the current study provides novel evidence that such a defect can impact economic decision making.

In summary, we have shown that VMPC is a critical brain area for normal decision making in the Ultimatum Game. It appears that proper modulation of emotional reactions, a faculty impaired by bilateral VMPC damage, is necessary for normal responses in situations of unfair treatment. More broadly, these data highlight the crucial role of emotional processes in situations of economic choice.

\section{References}

Anderson SW, Barrash J, Bechara A, Tranel D (2006) Impairments of emotion and real-world complex behavior following childhood- or adultonset damage to ventromedial prefrontal cortex. J Int Neuropsychol Soc 12:224-235.

Barrash J, Tranel D, Anderson SW (2000) Acquired personality disturbances associated with bilateral damage to the ventromedial prefrontal region. Dev Neuropsychol 18:355-381.

Beauregard M, Levesque J, Bourgouin P (2001) Neural correlates of conscious self-regulation of emotion. J Neurosci 21:165.

Bechara A (2004) Disturbances of emotion regulation after focal brain lesions. Int Rev Neurobiol 62:159-193.

Bechara A, Tranel D, Damasio H, Damasio AR (1996) Failure to respond autonomically to anticipated future outcomes following damage to prefrontal cortex. Cereb Cortex 6:215-225.

Berlin HA, Rolls ET, Kischka U (2004) Impulsivity, time perception, emotion, and reinforcement sensitivity in patients with orbitofrontal cortex lesions. Brain 127:1108-1126.

Blair RJR, Cipolotti L (2000) Impaired social response reversal: a case of acquired sociopathy. Brain 123:1122-1141.

Blumer D, Benson DF (1975) Personality changes with frontal and temporal lobe lesions. In: Psychiatric aspects of neurological disease (Benson DF, Blumer D, eds), pp 151-170. NewYork: Grune and Stratton.

Bolton GE, Zwick R (1995) Anonymity versus punishment in ultimatum bargaining. Games Economic Behav 10:95-121.

Burgess PW, Wood RL (1990) Neuropsychology of behaviour disorders following brain injury. In: Neurobehavioural sequelae of traumatic brain injury (Wood RL, ed), pp 110-133. New York: Taylor and Francis.

Calder AJ, Keane J, Manes F, Antoun N, Young AW (2000) Impaired recognition and experience of disgust following brain injury. Nat Neurosci 3:1077-1078.

Camille N, Coricelli G, Sallet J, Pradat-Diehl P, Duhamel JR, Sirigu A (2004) The involvement of the orbitofrontal cortex in the experience of regret. Science 304:1167-1170.

Damasio AR, Tranel D, Damasio H (1990) Individuals with sociopathic behavior caused by frontal damage fail to respond autonomically to social stimuli. Behav Brain Res 41:81-94.

Damasio AR, Grabowski TJ, Bechara A, Damasio H, Ponto LL, Parvizi J, Hichwa RD (2000) Subcortical and cortical brain activity during the feeling of self-generated emotions. Nat Neurosci 3:1049-1056.

Damasio H, Frank R (1992) Three-dimensional in vivo mapping of brain lesions in humans. Arch Neurol 49:137-143.

Damasio H, Tranel D, Grabowski T, Adolphs R, Damasio A (2004) Neural systems behind word and concept retrieval. Cognition 92:179-229.

Eslinger PJ, Damasio AR (1985) Severe disturbance of higher cognition after bilateral frontal lobe ablation: patient EVR. Neurology 35:1731-1741.

Frank RJ, Damasio H, Grabowski TJ (1997) Brainvox: an interactive, multimodal visualization and analysis system for neuroanatomical imaging. NeuroImage 5:13-30.

Grafman J, Schwab K, Warden D, Pridgen A, Brown HR, Salazar AM (1996) Frontal lobe injuries, violence, and aggression: a report of the Vietnam head injury study. Neurology 46:1231-1238.

Guth W, Schmittenberger R, Schwarze B (1982) An experimental analysis of ultimatum bargaining. J Econ Behav Organ 3:376.

Hanley JA, Negassa A, Edwardes MD, Forrester JE (2003) Statistical analysis of correlated data using generalized estimating equations: an orientation. Am J Epidemiol 157:364-375.

Kravitz D, Gunto S (1992) Decisions and recipients in ultimatum bargaining games. J Soc Econ 21:65-84.

Levesque J, Eugene F, Joanette Y, Paquette V, Mensour B, Beaudoin G, Leroux JM, Bourgouin P, Beauregard M (2003) Neural circuitry underlying voluntary suppression of sadness. Biol Psychiatry 53:502-510.

Ochsner KN, Bunge SA, Gross JJ, Gabrieli JD (2002) Rethinking feelings: an FMRI study of the cognitive regulation of emotion. J Cogn Neurosci 14:1215-1229.

Ochsner KN, Ray RD, Cooper JC, Robertson ER, Chopra S, Gabrieli JD, Gross 
JJ (2004) For better or for worse: neural systems supporting the cognitive down- and up-regulation of negative emotion. NeuroImage 23:483-499.

O’Doherty J, Kringelbach ML, Rolls ET, Hornak J, Andrews C (2001) Abstract reward and punishment representations in the human orbitofrontal cortex. Nat Neurosci 4:95-102.

Phan KL, Fitzgerald DA, Nathan PJ, Moore GJ, Uhde TW, Tancer ME (2005) Neural substrates for voluntary suppression of negative affect: a functional magnetic resonance imaging study. Biol Psychiatry 57:210-219.

Phillips ML, Young AW, Senior C, Brammer M, Andrew C, Calder AJ, Bullmore ET, Perrett DI, Rowland D, Williams SC, Gray JA, David AS (1997) A specific neural substrate for perceiving facial expressions of disgust. Nature 389:495-498.

Pillutla MM, Murnighan JK (1996) Unfairness, anger, and spite: emotional rejections of ultimatum offers. Org Behav Hum Dec Proc 68:208-224.
Rolls ET, Hornak J, Wade D, McGrath J (1994) Emotion-related learning in patients with social and emotional changes associated with frontal lobe damage. J Neurol Neurosurg Psychiatry 57:1518-1524.

Sanfey AG, Rillig JK, Aronson JA, Nystrom LE, Cohen JD (2003) The neural basis of economic decision-making in the ultimatum game. Science 300:1755-1758

Shallice T, Burgess PW (1991) Deficits in strategy application following frontal lobe damage in man. Brain 114:727-741.

Shiv B, Loewenstein G, Bechara A (2005) The dark side of emotion in decision-making: when individuals with decreased emotional reactions make more advantageous decisions. Cogn Brain Res 23:85-92.

Tranel D, Bechara A, Damasio H, Damasio AR (1998) Neural correlates of emotional imagery. Int J Psychophysiol 30:107.

van't Wout M, Kahn RS, Sanfey AG, Aleman A (2006) Affective state and decision-making in the ultimatum game. Exp Brain Res 169:564-568. 